\title{
A CONVERGENT FRAMEWORK FOR THE MULTICOMPONENT KP-HIERARCHY
}

\author{
G. F. HELMINCK AND G. F. POST
}

\begin{abstract}
In this paper we describe how to construct convergent solutions of the multicomponent KP-hierarchy, starting from a certain open subset of the Grassmann manifold of a special kind of Banach space, and derive an expression of its solutions in terms of Fredholm determinants. Further we show that the simplest nonscalar reduction of the present hierarchy leads to the AKNShierarchy.
\end{abstract}

\section{INTRODUCTION}

In $\S 1$ we introduce the multicomponent KP-hierarchy, following [U], and we show that in order to obtain solutions of this collection of nonlinear partial differential equations it suffices to solve a set of linear equations. $\S 2$ contains a description of the Grassmann manifold of a certain Banach space, which is the starting point of our construction. The actual construction of the solutions is given in $\S 3$. The scheme according to which we obtain solutions of the multicomponent KP-hierarchy is the same as that used in [Se] to construct solutions of the KP-hierarchy. $\S 4$ expresses the wave function in terms of $\tau$-functions. We conclude $(\S 5)$ with a discussion of reductions of the present hierarchy and give the relation with the AKNS-hierarchy.

\section{THE MULTICOMPONENT KP-HIERARCHY}

1.1. First we describe the framework in which the above-mentioned hierarchy is formulated. Let $B$ be an associative algebra over $\mathbb{C}$ with identity element and $\partial$ a $\mathbb{C}$-derivation of $B$. Following [Wi], we denote the algebra of "pseudodifferential operators with coefficients in $B$ " by $B\left[\xi, \xi^{-1}\right]$. Its elements are formal expressions,

$$
\sum_{j=-\infty}^{N} b_{j} \xi^{j}, \quad b_{j} \in B \text { for all } j \text { and } N \in \mathbb{Z},
$$

which are added componentwise and whose multiplication is based on the

Received by the editors March 9, 1989.

1980 Mathematics Subject Classification (1985 Revision). Primary 35Q20; Secondary 22E65, $14 \mathrm{M} 15$. 
following rules:

$$
\begin{array}{ll}
\xi^{i} \cdot \xi^{j}=\xi^{i+j} & \text { for all } i \text { and } j \text { in } \mathbb{Z}, \\
\xi^{m} \cdot b=\sum_{j=0}^{\infty}\left(\begin{array}{c}
m \\
j
\end{array}\right) \partial^{j}(b) \xi^{m-j} & \text { for all } m \text { in } \mathbb{Z} \text { and } b \in B .
\end{array}
$$

If $P=\sum b_{j} \xi^{j}$ is any element of $B\left[\xi, \xi^{-1}\right]$, then we write $P_{+}$for the differential operator part of $P$ and we denote $P-P_{+}$by $P_{-}$, i.e.,

$$
P_{+}=\sum_{i \geq 0} b_{i} \xi^{i} \text { and } P_{-}=\sum_{i<0} b_{i} \xi^{i}
$$

The subalgebra of the differential operators in $B\left[\xi, \xi^{-1}\right]$, i.e., those $P$ for which $P=P_{+}$, is denoted by $B[\xi]$.

1.1.1. Remark. One easily verifies that a $K=\sum_{i \leq N} k_{i} \xi^{i}$ in $B\left[\xi, \xi^{-1}\right]$, with $k_{N} \neq 0$, is invertible if and only if $k_{N}$ is invertible in $B$.

1.2. The elements of $B\left[\xi, \xi^{-1}\right]$ can be interpreted as operators by letting them act through multiplication on $B\left[\xi, \xi^{-1}\right]$ itself. This leads one to the definition of the following $B\left[\xi, \xi^{-1}\right]$-module: Let $M(1)$ be the space of all power series in $\lambda$ with coefficients in $B$ which have a "pole at infinity," i.e., all sums $\sum_{i=-\infty}^{N} b_{i} \lambda^{i}$, with $b_{i} \in B$. Its $B\left[\xi, \xi^{-1}\right]$-module structure is an algebra extension of the following actions:

$$
\begin{aligned}
b \cdot \sum_{i} b_{i} \lambda^{i} & =\sum_{i} b b_{i} \lambda^{i}, \quad \text { with } b \in B, \\
\xi \cdot \sum_{i} b_{i} \lambda^{i} & =\sum_{i} \partial\left(b_{i}\right) \lambda^{i}+\sum_{i} b_{i} \lambda^{i+1}, \\
\xi^{-1} \cdot \sum_{i} b_{i} \lambda^{i} & =\sum_{j=0}^{\infty} \sum_{i}(-1)^{j} \partial^{j}\left(b_{i}\right) \lambda^{i-j-1} .
\end{aligned}
$$

Clearly $M(1)$ is a free $B\left[\xi, \xi^{-1}\right]$-module with generator 1 . The $B\left[\xi, \xi^{-1}\right]$ module structure on a space of power series in $\lambda$ with another prescribed behaviour around infinity is trivially carried over from $M(1)$. To be more precise, let $g(\lambda)$ be a power series in $\lambda$ with coefficients in $B$. Denote $\{f(\lambda) g(\lambda) \mid f(\lambda) \in$ $M(1)\}$ by $M(g(\lambda))$. Then $M(g(\lambda))$ becomes a $B\left[\xi, \xi^{-1}\right]$-module by simply putting

$$
P(f(\lambda) g(\lambda))=(P \cdot f(\lambda)) g(\lambda) \text { for each } P \text { in } B\left[\xi, \xi^{-1}\right] .
$$

Hence each $M(g(\lambda))$ is a free $B\left[\xi, \xi^{-1}\right]$-module with generator $g(\lambda)$. This fact is used to obtain equations for operators in $B\left[\xi, \xi^{-1}\right]$ by their action on elements of $M(g(\lambda))$.

1.3. To give the form of the hierarchy we now specify $B$. The algebra $B$ consists of the square matrices of size $m$ with entries from a commutative $\mathbb{C}$ algebra $R$. We assume that $R$ contains $\mathbb{C}\left[t_{i, \alpha}\right]$, as a subalgebra, where $i \in \mathbb{N}$ 
and $1 \leq \alpha \leq m$, and that there exist for all those $i$ and $\alpha$ derivations $\partial_{i, \alpha}$ of $R$ which extend the partial derivative w.r.t. $t_{i, \alpha}$ from $\mathbb{C}\left[t_{i, \alpha}\right]$ to $R$. These derivations of $R$ extend in a natural way to derivations of $B$ and they are denoted by the same symbol. For each $i$ and $\alpha$, we let $\partial_{i, \alpha}$ act coefficientwise on $B\left[\xi, \xi^{-1}\right]$ and $M(1)$. Of the derivation $\partial$ of $B$ we require for the moment only that it commutes with all the $\partial_{i, \alpha}$. We write $E_{\alpha}$ for the element of $B$, which has a 1 on the $(\alpha, \alpha)$-entry and zeros elsewhere. We consider operators $L$ and $U_{\alpha}, 1 \leq \alpha \leq m$, in $B\left[\xi, \xi^{-1}\right]$ of the form

$$
L=\xi+\sum_{j<0} l_{j} \xi^{j} \text { and } U_{\alpha}=E_{\alpha}+\sum_{j<0} u_{j, \alpha} \xi^{j}
$$

Now the multicomponent KP-hierarchy consists of an infinite set of differential equations for the coefficients $l_{j}$ and $u_{j, \alpha}$, which are conveniently formulated in terms of the operators $L$ and $U_{\alpha}$. These equations are

$$
\begin{gathered}
\partial_{i, \alpha}(L)=\left[\left(L^{i} U_{\alpha}\right)_{+}, L\right], \\
\partial_{i, \alpha}\left(U_{\beta}\right)=\left[\left(L^{i} U_{\alpha}\right)_{+}, U_{\beta}\right] .
\end{gathered}
$$

Since the left-hand sides of (1.3.1) and (1.3.2) contain only powers of $\xi$ with negative exponents, it would be nice if this would hold for the right-hand sides too. This is the case if $L$ and the $U_{\beta}$ commute with the $L^{i} U_{\alpha}$, for then we have $\left[\left(L^{i} U_{\alpha}\right)_{+}, L\right]=-\left[\left(L^{i} U_{\alpha}\right)_{-}, L\right]$ and $\left[\left(L^{i} U_{\alpha}\right)_{+}, U_{\beta}\right]=-\left[\left(L^{i} U_{\alpha}\right)_{-}, U_{\beta}\right]$. Therefore we assume that the $U_{\alpha}$ commute among each other and with $L$. The KP-hierarchy corresponds to the case $m=1$ and $U_{\alpha}=1$.

1.4. An essential step in the construction of solutions of (1.3.1) and (1.3.2) is to view them as compatibility conditions for a set of linear equations. Before giving them, we introduce for each $i \in \mathbb{N}$ and $1 \leq \alpha \leq m$ an endomorphism $\tilde{\partial}_{i, \alpha}$ of $M(g(\lambda))$ by

$$
\tilde{\partial}_{i, \alpha}(f(\lambda) g(\lambda))=\left\{\partial_{i, \alpha}(f(\lambda))+f(\lambda) E_{\alpha} \lambda^{i}\right\} g(\lambda) .
$$

The property of $\tilde{\partial}_{i, \alpha}$ one needs is that for all $P$ in $B\left[\xi, \xi^{-1}\right]$ and $\underline{m}$ in $M(g(\lambda))$,

$$
\tilde{\partial}_{i, \alpha}(P \cdot \underline{m})=\partial_{i, \alpha}(P) \cdot \underline{m}+P \cdot \tilde{\partial}_{i, \alpha}(\underline{m}) .
$$

If we choose $g(\lambda)$ such that applying $\partial_{i, \alpha}$ termwise to $g(\lambda)$ results in $E_{\alpha} \lambda^{i} g(\lambda)$, then $\tilde{\partial}_{i, \alpha}$ coincides with applying $\partial_{i, \alpha}$ termwise to the series $f(\lambda) g(\lambda)$. Such a choice is available in the present context, for if we take

$$
g(\lambda)=\exp \left(\operatorname{diag}\left(\sum_{i \geq 1} t_{i, 1} \lambda^{i}, \ldots, \sum_{i \geq 1} t_{i, m} \lambda^{i}\right)\right),
$$

then the entries of the coefficients of $g(\lambda)$ belong to $\mathbb{C}\left[t_{i, \alpha}\right]$. This is the $g(\lambda)$ that we choose in practice. Let $L$ and the $U_{\alpha}$ be as above. Consider now for 
$w$ in $M(g(\lambda))$ the equations

$$
\begin{gathered}
L(w)=\lambda w, \\
U_{\alpha}(w)=w E_{\alpha}, \\
\tilde{\partial}_{i, \alpha}(w)=\left(L^{i} U_{\alpha}\right)_{+}(w) .
\end{gathered}
$$

A nonzero $w$ satisfying these equations is called a wave function corresponding to $L$ and the $U_{\alpha}$. Clearly if $r_{1}, \ldots, r_{m}$ belong to $\mathbb{Z}$ and $w$ is a wave function corresponding to $L$ and the $U_{\alpha}$, then so is $w \operatorname{diag}\left(\lambda^{r_{1}}, \ldots, \lambda^{r_{m}}\right)$. Hence we may assume that a wave function has the form $w=\left\{\sum_{i \leq 0} w_{i} \lambda^{i}\right\} g(\lambda)$, with $w_{0} \neq 0$. If we associate to $w$ a $\widehat{W}=\sum_{i \leq 0} w_{i} \xi^{i}$ in $B\left[\xi, \xi^{-1}\right]$, then the equations (1.4.1), (1.4.2), and (1.4.3) amount to the following relations in $B\left[\xi, \xi^{-1}\right]$ :

$$
\begin{gathered}
L \widehat{W}=\widehat{W} \xi \\
U_{\alpha} \widehat{W}=\widehat{W} E_{\alpha}, \\
\partial_{i, \alpha}(\widehat{W})+\widehat{W} E_{\alpha} \xi^{i}=\left(L^{i} U_{\alpha}\right)_{+} \widehat{W} .
\end{gathered}
$$

If one applies $\tilde{\partial}_{i, \beta}$ to both sides of (1.4.1) and (1.4.2) and substitutes (1.4.3) into it, then, after translating the result back to $B\left[\xi, \xi^{-1}\right]$, one obtains

$$
\partial_{i, \beta}(L) \widehat{W}=\left(L^{i} U_{\beta}\right)_{+} \widehat{W} \xi-L\left(L^{i} U_{\beta}\right)_{+} \widehat{W}
$$

and

$$
\partial_{i, \beta}\left(U_{\alpha}\right) \widehat{W}=\left(L^{i} U_{\beta}\right)_{+} \widehat{W} E_{\alpha}-U_{\alpha}\left(L^{i} U_{\beta}\right)_{+} \widehat{W} .
$$

If $\widehat{W}$ is invertible, then these relations imply that $L$ and the $U_{\alpha}$ satisfy the equations of the multicomponent KP-hierarchy and for that it is sufficient that $w_{0}$ is invertible, see (1.1.1). In that case we see moreover that

$$
L=\widehat{W} \xi \widehat{W}^{-1} \text { and } U_{\alpha}=\widehat{W} E_{\alpha} \widehat{W}^{-1} .
$$

Hence $L$ and the $U_{\alpha}$ are obtained by "dressing" the trivial solution $\left(\xi, E_{\alpha}\right)$. Note that all such defined $L$ and $U_{\alpha}$ commute. Conversely, given an operator $W$ of the form $W=\sum_{i \leq 0} w_{i} \xi^{i}$, with $w_{0}=\mathrm{Id}$, one can then define $L$ and the $U_{\alpha}$ by these formulas and, in order that they satisfy the equations of the multicomponent KP-hierarchy, it is sufficient to show that $\sum_{i \leq 0} w_{i} \lambda^{i}$ satisfies (1.4.3). This is the procedure that we will follow in $\S 3$. Solutions obtained in this way satisfy $\left[L, U_{\alpha}\right]=\left[U_{\alpha}, U_{\beta}\right]=0, U_{\alpha} U_{\beta}=\delta_{\alpha \beta} U_{\beta}$, and $\sum_{\alpha=1}^{m} U_{\alpha}=$ Id . These are the assumptions imposed on $L$ and the $U_{\alpha}$ in $[U]$.

1.5. The equations (1.3.1) and (1.3.2) possess a certain scaling stability. We will explain here what we mean by this. In order that the following computations make sense, we will assume that $R$ consists of functions in the parameters $t_{i, \alpha}$ and that each $\partial_{i, \alpha}$ is differentiation w.r.t. $t_{i, \alpha}$. Since all solutions to be constructed are of this type, this is no serious restriction. 
Let $c$ be in $\mathbb{C}^{*}$. For each $u$ in $R$ we put

$$
\tilde{u}\left(t_{i, \alpha}\right)=u\left(c^{i} t_{i, \alpha}\right)
$$

and likewise, for $b=\left(b_{i j}\right) \in B,(\tilde{b})_{i j}=\tilde{b}_{i j}$. We write $\widetilde{R}$, resp. $\widetilde{B}$, for $\{\tilde{u} \mid u \in R\}$, resp. $\{\tilde{b} \mid b \in B\}$. Now let $\left(L, U_{\alpha}\right)$ as in (1.3) be a solution of the multicomponent KP-hierarchy. Consider

$$
\widetilde{L}=\xi+\sum_{i<0} c^{-i+1} \tilde{l}_{i} \xi^{i} \text { and } \tilde{U}_{\alpha}=E_{\alpha}+\sum_{j<0} c^{-j} \tilde{u}_{j, \alpha} \xi^{j}
$$

Then $\widetilde{L}$ and $\widetilde{U}_{\alpha}$ belong to $\widetilde{B}\left[\xi, \xi^{-1}\right]$ and we call them the scalings of $\left(L, U_{\alpha}\right)$ by a factor $c$. We claim that $\left(\widetilde{L}, \widetilde{U}_{\alpha}\right)$ are again a solution of the multicomponent KP-hierarchy. This is the stability we had in mind. This statement is most easily seen if one introduces the $\operatorname{End}\left(\mathbb{C}^{m}\right)$-algebra $\underline{B}$ generated by the formal noncommutative symbols $\underline{\partial^{k}\left(l_{i}\right)}$ and $\underline{\partial^{r}\left(u_{j, \alpha}\right)}$, with $k, r \geq 0, i, j<0$, and $1 \leq \alpha \leq m$. Clearly we can speak of $\underline{B}\left[\xi, \xi^{-1}\right]$ and we put a grading on this algebra by prescribing it on its generators. We give $\partial^{k}\left(l_{i}\right)$ degree $k-i+1, \partial^{r}\left(u_{j, \alpha}\right)$ degree $-j+r$, and $\xi^{r}$ degree $r$. An element $\overline{\sum_{i \leq N_{i}}} a_{i} \xi^{i}$ in $\underline{B}\left[\xi, \xi^{-1}\right]$ is called homogeneous of degree $n$ if the degree of $a_{i} \xi^{i}$ is $n$ for all $i$. In particular $\underline{L}=\xi+\sum_{i<0} \underline{l}_{i} \xi^{i}$ is homogeneous of degree one and $\underline{U_{\alpha}}=E_{\alpha}+\sum_{j<0} u_{j, \alpha} \xi^{j}$ is homogeneous of degree zero. Since homogeneity is preserved under multiplication, we get

$$
\left[\left(\underline{L}^{i} \underline{U_{\alpha}}\right)_{+}, \underline{L}\right]=\sum_{j>0} A_{i j} \xi^{-j}
$$

and

$$
\left[\left(\underline{L}^{i} \underline{U_{\alpha}}\right)_{+}, \underline{U_{\beta}}\right]=\sum_{j>0} C_{i j} \xi^{-j},
$$

with $A_{i j}$, resp. $C_{i j}$, homogeneous polynomial expressions in the $\partial^{r}\left(l_{i}\right)$ and $\partial^{s}\left(u_{j, \alpha}\right)$ of degree $i-j+1$, resp. $i-j$. Then (1.3.1) and (1.3.2) amount to the following relations for the $l_{i}$ and $u_{j, \alpha}$ :

$$
\begin{gathered}
\partial_{i, \alpha}\left(l_{j}\right)=A_{i j}\left(\partial^{r}\left(l_{s}\right), \partial^{p}\left(u_{t, \gamma}\right)\right), \\
\partial_{i, \alpha}\left(u_{j, \beta}\right)=C_{i j}\left(\partial^{r}\left(l_{s}\right), \partial^{p}\left(u_{t, \gamma}\right)\right) .
\end{gathered}
$$

Thus we must show the same relations for $l_{j}$ replaced by $c^{-j+1} \tilde{l}_{j}$ and $u_{j, \beta}$ replaced by $c^{-j} \tilde{u}_{j, \beta}$. But

$$
\begin{aligned}
\partial_{i, \alpha}\left(c^{-j+1} \tilde{l}_{j}\right) & =c^{i+1-j}\left(\partial_{i, \alpha}\left(l_{j}\right)\right)^{\sim}=c^{\operatorname{degree}\left(A_{i j}\right)} \tilde{A}_{i j} \\
& =A_{i j}\left(\partial^{r}\left(c^{-s+1} \tilde{l}_{s}\right), \partial^{p}\left(c^{-t} \tilde{u}_{t, \gamma}\right)\right),
\end{aligned}
$$




$$
\begin{aligned}
\partial_{i, \alpha}\left(c^{-j} \tilde{u}_{j}\right) & =c^{i-j}\left(\partial_{i, \alpha}\left(u_{j, \beta}\right)\right)^{\sim}=c^{\operatorname{degree}\left(C_{i j}\right)} \widetilde{C}_{i j} \\
& =C_{i j}\left(\partial^{r}\left(c^{-s+1} \tilde{l}_{s}\right), \partial^{p}\left(c^{-t} \tilde{u}_{t, \gamma}\right)\right),
\end{aligned}
$$

and this proves the assertion.

\section{ThE GRASSMANN MANIFOLD}

2.1. In this section we recall the definition and some properties of the Grassmann manifold of a certain Banach space $H$, such as was introduced in [He]. First of all $H$ is a subspace of the space of all functions from $\mathbb{Z}$ to $\mathbb{C}$. Denote the map which sends $i$ to one and all other integers to zero by $v_{i}$. Hence all elements of $H$ have a decomposition

$$
\sum_{i \in \mathbb{Z}} a_{i} v_{i}, \quad a_{i} \in \mathbb{C},
$$

into the basic elements $v_{i}$. For any $S \subseteq \mathbb{Z}$, we write $H_{s}$ for

$$
\left\{h=\sum a_{i} v_{i} \mid h \in H, a_{i}=0 \forall i \notin S\right\} .
$$

In particular, we combine this notation with the following two: $\geq 0=\{n \mid n \in \mathbb{Z}$, $n \geq 0\}$ and $<0=\{m \mid m \in \mathbb{Z}, m<0\}$. Further we make the following convention: if we use the word "natural" as an adjective for a map from a subspace $W$ to $H$ to some $H_{s}$, then we mean the map

$$
w=\sum c_{i} v_{i} \rightarrow \sum_{i \in S} c_{i} v_{i}
$$

We assume that the normed space $H$ has the following properties:

2.1.1. $H$ contains all the $v_{i}, i \in \mathbb{Z}$, and all the natural projections $H \rightarrow H_{\{i\}}$ are continuous.

2.1.2. Let $p_{+}$be the prescription $\sum a_{i} v_{i} \rightarrow \sum_{i \geq 0} a_{i} v_{i}$. Then $p_{+}$maps $H$ continuously onto $H_{\geq 0}$. In particular, we have the decomposition $H=$ $H_{>0} \oplus H_{<0}$.

2.1.3. The prescription $\Lambda: \sum_{i \in \mathbb{Z}} a_{i} v_{i} \rightarrow \sum_{i \in \mathbb{Z}} a_{i} v_{i+1}$ defines a bounded endomorphism of $H$ with operator norm $\|\Lambda\|$.

2.1.4. The $v_{i}, i \in \mathbb{Z}$, form a topological base of $H$, i.e., if $v=\sum a_{i} v_{i} \in H$ then $v=\lim _{M \rightarrow \infty} \sum_{i=-M}^{M} a_{i} v_{i}$.

2.2. Remarks. (a) Examples of Banach spaces satisfying all these properties are the $l^{p}(\mu), 1 \leq p<\infty$ and $\mu$ a positive measure on $\mathbb{Z}$ satisfying $\mu(i+1) \leq$ $M \mu(i)$, for some constant $M$ and all $i$ in $\mathbb{Z}$.

(b) Thanks to property 2.1 .3 , each power series $\sum_{i \geq 0} a_{i} z^{i}, a_{i} \in \mathbb{C}$, with radius of convergence greater than $\|\Lambda\|$ determines an endomorphism $\sum_{i \geq 0} a_{i} \Lambda^{i}$ of $H$. We will use the notation $\Gamma(\Lambda)$ for $\left\{\gamma \mid \gamma \in \operatorname{Aut}(H), \gamma=\exp \left(\sum_{i \geq 1} a_{i} \Lambda^{i}\right)\right.$ with the $a_{i}$ coefficients of such a power series\}.

(c) $H$ can be realized as a certain space of complex power series in $\lambda$ by mapping $\sum a_{i} v_{i}$ to $\sum a_{i} \lambda^{i}$ and simply transferring the norm. This realization 
was used in the case of the KP-hierarchy. Here we need a vector version of this realization (see 3.1).

(d) Since $H_{\geq 0}$ possesses the approximation property, we can speak of the determinant for each operator in $\operatorname{End}\left(H_{\geq 0}\right)$ of the form "Id + nuclear" (see $[\mathrm{He}])$.

2.3. For any subspace $W$ of $H$ we denote the canonical embedding of $W$ into $H$ by $i_{w}$. Let $\operatorname{Gr}_{k}(H)$ be the collection of closed subspaces $W$ in $H$ such that $p_{+} \circ i_{w}$ is a Fredholm operator of index $k$. As in [He] we introduce

2.3.1. Definition. The Grassmann manifold of $H$ is the collection of all subspaces $\mathrm{Gr}_{k}(H), k \in \mathbb{Z}$.

Our object of interest is the space $\mathrm{Gr}_{0}(H)$, which we also denote by Gr. Recall that the Grassmann manifold consisting of all $n$-dimensional subspaces of $\mathbb{C}^{n+m}$ is isomorphic to the quotient of the space of complex $(n+m) \times n$ matrices of rank $n$ under the natural right action of $\mathrm{Gl}_{n}(\mathbb{C})$ or, to say it in other words, it is the space of all embeddings of $\mathbb{C}^{n}$ into $\mathbb{C}^{n+m}$ divided by the group of automorphisms of $\mathbb{C}^{n}$. An analogue of this description exists also for Gr. Consider the space $\mathbf{P}$ of all continuous embeddings $w$ of $H_{\geq 0}$ into $H$, which satisfy

2.3.2. $w_{+}=p_{+} \circ w$ has the form "identity + a nuclear operator."

Since nuclear operators are compact, $w_{+}$is a Fredholm operator of index zero and, in particular, the image of $w$ is a closed subspace of $H$. Clearly $\operatorname{im}(w)$ belongs to $\mathrm{Gr}$. Since finite-dimensional operators are nuclear, each $W$ in $\mathrm{Gr}$ occurs as the image of an element of $\mathbf{P}$. Two elements $w_{1}$ and $w_{2}$ of $\mathbf{P}$ have the same image if and only if they differ by an element of $\mathbf{T}$, i.e., $w_{2}=w_{1} \circ t, t \in \mathbf{T}$, where $\mathbf{T}$ is the group of automorphisms of $H_{\geq 0}$ which have the form "identity + nuclear." Hence under the projection $\pi: \mathbf{P} \rightarrow \mathrm{Gr}$, $\pi(w)=\operatorname{im}(w)$, we get an identification of $\mathrm{Gr}$ with $\mathbf{P} / \mathbf{T}$.

The topology of $\mathrm{Gr}$ is derived from that of $\mathbf{P}$ : a bases of neighbourhoods of $w$ in $\mathbf{P}$ consists of

$$
\left\{p \mid p \in \mathbf{P},\left\|p_{+}-w_{+}\right\|_{1}<\varepsilon \text { and }\left\|p_{-}-w_{-}\right\|<\varepsilon, \varepsilon>0\right\},
$$

where $\|-\|_{1}$ is the Schatten-norm on the space of nuclear operators, $\|-\|$ denotes the operator norm and $\left(\begin{array}{l}w_{+} \\ w_{-}\end{array}\right)$, resp. $\left(\begin{array}{l}p_{+} \\ p_{-}\end{array}\right)$, are the decompositions of $w$ and $p$ w.r.t. $H=H_{\geq 0} \oplus H_{<0}$. T becomes a topological group if we take $\{t \mid t \in$ $\left.\mathrm{T},\|t-\mathrm{id}\|_{1}<\varepsilon\right\}, \varepsilon>0$, as the neighbourhood base of the identity. It is not difficult to see that the natural right action of $\mathbf{T}$ on $\mathbf{P}$ is continuous. We then put the quotient topology on Gr.

Apart from the description given above of $\mathrm{Gr}$, we can also see it as an orbit of a certain group of automorphisms of $H$ under its natural action. To be more precise, let $G_{n}$ be the group of bounded automorphisms of $H$ which have, with 
respect to the decomposition $H=H_{\geq 0} \oplus H_{<0}$, the form

$$
\left\{\left(\begin{array}{ll}
a & b \\
c & d
\end{array}\right) \mid \begin{array}{l}
a \text { and } d \text { are Fredholm operators with } \\
\text { index }(a)=\text { index }(d)=0, c \text { is bounded } \\
\text { and } b \text { is a nuclear operator. }
\end{array}\right\}
$$

$G_{n}$ acts on $\mathrm{Gr}$ by its natural action on $H$ and it is not difficult to see that this action is transitive. Hence we have $\mathrm{Gr}$ as the quotient $G_{n} / B_{n}$, where $B_{n}$ is the stabilizer of $H_{\geq 0}$. Further $G_{n}$ is easily seen to act continuously on $\mathrm{Gr}$ if we take the following as a neighbourhood base of the identity in $G_{n}$ :

$$
\left\{g \mid g \in G_{n}, g=\left[\begin{array}{ll}
a & b \\
c & d
\end{array}\right],\|a-\mathrm{id}\|<\varepsilon,\|d-\mathrm{id}\|<\varepsilon,\|c\|<\varepsilon,\|b\|_{1}<\varepsilon\right\} .
$$

It is shown in [He] that the elements of $\Gamma(\Lambda)$ are examples of automorphisms lying in $B_{n}$ and the realizations $\mathbf{P} / \mathbf{T}$ and $G_{n} / B_{n}$ of $\mathrm{Gr}$ are chosen to include operators of this form.

2.4. On Gr we define the determinant bundle Det and its dual Det ${ }^{*}$ as the orbit spaces corresponding respectively to the following actions of $\mathbf{T}$ on $\mathbf{P} \times \mathbb{C}$ :

$$
\begin{gathered}
(w, \lambda) \rightarrow\left(w \circ t, \lambda \operatorname{det}(t)^{-1}\right), \\
(w, \lambda) \rightarrow(w \circ t, \lambda \operatorname{det}(t)) .
\end{gathered}
$$

Here $w$ belongs to $\mathbf{P}, t$ to $\mathbf{T}$, and $\lambda$ lies in $\mathbb{C}$. From the second action it will be clear that we can define a section $\sigma$ of Det ${ }^{*}$ by

$$
\sigma(\pi(w))=\left(w, \operatorname{det}\left(w_{+}\right)\right) .
$$

Since it is not clear how to define an action of $G_{n}$ on Det or Det ${ }^{*}$ we pass to an extension $G$ of $G_{n}$. First we note that for each $g=\left[\begin{array}{ll}a & b \\ c & d\end{array}\right]$ in $G_{n}$ there exists an automorphism $q$ of $H_{\geq 0}$ such that $a q^{-1}-\mathrm{id}$ is finite dimensional. For $G$ we now take the collection of pairs $(g, q)$ with $g$ as above in $G_{n}$ and $q$ an automorphism of $H_{\geq 0}$ such that $a q^{-1}-$ id is nuclear. This set forms a group w.r.t. the natural composition. Since $\operatorname{det}(t)=\operatorname{det}\left(q t q^{-1}\right)$ for each $t$ in $\mathbf{T}$ and for each $q$ in $\operatorname{Aut}\left(H_{\geq 0}\right)$, we can define an action of $G$ on Det ${ }^{*}$ by

$$
(g, q) \cdot(w, \lambda)=\left(g \circ w \circ q^{-1}, \lambda\right) .
$$

$G$ becomes a continuous transformation group of Det $^{*}$ if we equip it with the topology generated by

$$
\left.\left\{\left(\begin{array}{ll}
a & b \\
c & d
\end{array}\right), q\right) \in G \mid \begin{array}{l}
\|a-\mathrm{id}\|<\varepsilon,\|d-\mathrm{id}\|<\varepsilon,\|q-\mathrm{id}\|<\varepsilon, \\
\left\|a q^{-1}-\mathrm{id}\right\|_{1}<\varepsilon,\|b\|_{1}<\varepsilon,\|c\|<\varepsilon
\end{array}\right\} .
$$

Note that there is a natural way to embed the group $B_{n}$ into $G$, namely

$$
\left(\begin{array}{ll}
a & b \\
0 & d
\end{array}\right) \rightarrow\left(\left(\begin{array}{ll}
a & b \\
0 & d
\end{array}\right), a\right)
$$


This will be assumed from now on. We conclude this section with defining for each $w$ in $\mathbf{P}$ a function $\tau_{w}: G \rightarrow \mathbb{C}$ by

$$
\tau_{w}\left((g, q)^{-1}\right)=\operatorname{det}\left(\left(g \circ w \circ q^{-1}\right)_{+}\right) .
$$

The restriction of $\tau_{w}$ to $B_{n}$ measures the failure of $B_{n}$-equivariance of $\sigma$ at the point $\pi(w)$ of $\mathrm{Gr}$. Moreover its zero-set forms the boundary of the domain to which the solutions (constructed below) of the hierarchy have an analytic continuation.

2.5. We introduce here the Banach spaces needed for the multicomponent case. Take $m$ copies $H^{(i)}, 1 \leq i \leq m$, of the space $H$ from (2.1). Denote their basic elements by $v_{k}^{(i)}, k \in \mathbb{Z}$ and $1 \leq i \leq m$. Consider the space $\mathbf{K}=$ $\bigoplus_{i=1}^{m} H^{(i)}$ with the sum norm and the basic elements $e_{i-k-m-1}=v_{k}^{(i)}, k \in \mathbb{Z}$ and $1 \leq i \leq m$. K satisfies the conditions (2.1.1), (2.1.2), (2.1.3), and (2.1.4) so that we can speak of $\operatorname{Gr}_{0}(\mathbf{K})$ and the dual of the determinant bundle over this space. We assume that all the $H^{(i)}$ are realized as power series in $\lambda$ as indicated in $2.2(\mathrm{c})$. Hence the elements of $\mathbf{K}$ are realized as power series in $\lambda$ with coefficients in $\mathbb{C}^{m}$ whose elements are written as rows. E.g. the elements $v_{k}^{(i)}$ correspond to $f_{i} \lambda^{k}$, with $f_{i}$ the standard basic vector of $\mathbb{C}^{m}$ with a one in the $i$ th place and zeros elsewhere. We embed $\operatorname{End}\left(\mathbb{C}^{m}\right)$ into End $\mathbf{K}$ in the following way: if $A=\left(a_{i j}\right) \in \operatorname{End}\left(\mathbb{C}^{m}\right)$ then the operator

$$
h=\left(k_{1}, \ldots, k_{m}\right) \rightarrow h A
$$

corresponds to $A$ with $h_{i} \in H$. We write $\underline{\Lambda}$ for the endomorphism $\operatorname{diag}(\Lambda, \ldots, \Lambda)$ of $\mathbf{K}$. Then we can define the operator $\sum_{i \geq 0} A_{i} \underline{\Lambda}^{i}$ for each series $A_{i}$ in $\operatorname{End}\left(\mathbb{C}^{m}\right)$ such that $\sum_{i \geq 0}\left\|A_{i}\right\||\underline{\Lambda}|^{i}<\infty$. The relevant flows on $\mathrm{Gr}_{0}(\mathbf{K})$ for the multicomponent KP-hierarchy are of this form. Namely let $\Gamma$ be the $m$-fold product of the $\Gamma(\Lambda)$. If $\gamma=\left(\gamma_{1}, \ldots, \gamma_{m}\right) \in \Gamma$, with $\gamma_{\alpha}=\exp \left(\sum_{j \geq 1} t_{j \alpha} \Lambda^{j}\right)$, then it acts on $\mathbf{K}$ by

$$
\left(h_{1}, \ldots, h_{m}\right) \rightarrow\left(h_{1} \exp \left(\sum_{j \geq 1} t_{j 1} \lambda^{j}\right), \ldots, h_{m} \exp \left(\sum t_{j m} \lambda^{j}\right)\right)
$$

In other words, $\gamma$ acts as $\exp \left(\sum_{j \geq 1} \operatorname{diag}\left(t_{j 1}, \ldots, t_{j m}\right) \underline{\Lambda}^{j}\right)$. Moreover these operators belong to $G_{n}$.

Next we want to introduce a "scaling" on the space $\mathrm{Gr}_{0}(\mathbf{K})$. Here we restrict attention to those $c$ in $\mathbb{C}^{*}$ for which

$$
\sum_{i \leq 0} w_{i} \lambda^{i} \rightarrow \sum_{i \leq 0} c^{-i} w_{i} \lambda^{i}, \quad w_{i} \in \mathbb{C}^{m}
$$

defines a bounded operator from $\mathbf{K}_{\leq 0}$ to $\mathbf{K}_{\leq 0}$. Clearly such $c$ satisfy $|c| \leq 1$. Every $w$ in $\mathbf{P}$ can, thanks to $(2.1 . \overline{4})$, be uniquely written in the form

$$
\sum_{i \geq 0} v_{i} \lambda^{i} \rightarrow \sum_{i \geq 0} \sum_{j \in \mathbb{Z}} v_{i} w_{j i} \lambda^{i}
$$


with $w_{j i} \in \operatorname{End}\left(\mathbb{C}^{m}\right)$ and $v_{i} \in \mathbb{C}^{m}$ for all $i$ and $j$. As in the one-dimensional case one shows that one may assume that the $w_{i j}$ satisfy the additional conditions $w_{j i}=0$ for all $j>i$ and there is a $N>0$ such that $w_{i i}=\operatorname{Id}$ for all $i \geq N$ and $w_{j i}=0$ for all $j \neq i, i, j \geq N$. Now let $R_{c}$ be the "scaling" prescription

$$
\sum_{i \in \mathbb{Z}} w_{i} \lambda^{i} \rightarrow \sum_{i \in \mathbb{Z}} w_{i} c^{-i} \lambda^{i}
$$

If one starts with a general power series in $\mathbf{K}$, then applying $R_{c}$ does not lead to an element in $\mathbf{K}$. However if we consider elements $\sum_{i \leq N} w_{i} \lambda^{i}$ in $K$, then (2.5.1) tells you that $R_{c}\left(\sum w_{i} \lambda^{i}\right)$ is again in $K$. As we have seen above, every $W$ in $\mathrm{Gr}_{0}(\mathbf{K})$ has a topological basis of this form. Now the scaling of $W$ by the factor $c, R_{c} W$, is by definition the closure of the span of the $R_{c}$-transforms of the elements in such a basis. $R_{c} W$ belongs again to $\mathrm{Gr}_{0}(\mathbf{K})$, since it is the image of the embedding

$$
\sum_{i \geq 0} v_{i} \lambda^{i} \rightarrow \sum_{i \geq 0} \sum_{j \in \mathbb{Z}} v_{i}\left(w_{j i} c^{i-j}\right) \lambda^{j},
$$

where $w_{j i}$ satisfies the assumptions above and thus belongs to $\mathbf{P}$.

\section{THE CONSTRUCTION OF THE SOLUTIONS}

3.1. The starting point of our construction is the space $\mathrm{Gr}=\mathrm{Gr}_{0}(\mathbf{K})$. For each $W$ in $\mathrm{Gr}$, consider the subset $\Gamma^{W}$ of $\Gamma$, defined by

$$
\begin{aligned}
\Gamma^{W} & =\left\{\gamma \mid \gamma \in \Gamma, \text { the natural projection } W \gamma^{-1} \rightarrow \mathbf{K}_{\geq 0} \text { is an isomorphism }\right\} \\
& =\left\{\gamma \mid \gamma \in \Gamma, \tau_{w}(\gamma) \neq 0 \text { for some } w \text { in } \mathbf{P} \text { with } \pi(w)=W\right\} .
\end{aligned}
$$

From this last equality it is clear that $\Gamma^{W}$ is an open subset of $\Gamma$. However, contrary to the case $m=1$, for $m \geq 2$ there are always subspaces $W$ in Gr such that $\Gamma^{W}$ is empty. Take, e.g. for each $i$ in $\{1, \ldots, m\}$, a $W_{i}$ in $\mathrm{Gr}_{k_{i}}(H)$, where not all $k_{i}$ are zero and $\sum_{i=1}^{m} k_{i}=0$. Then $W=\bigoplus_{i=1}^{m} W_{i}$ lies in $\mathrm{Gr}=\mathrm{Gr}_{0}(\mathbf{K})$ but $\Gamma^{W}$ is empty. Nevertheless the collection $\mathbf{O}$ consisting of all $W$ in Gr with $\Gamma^{W}$ nonempty, is a nonvoid open subset of Gr. For each $W$ in $\mathbf{O}$ we will construct a solution of the hierarchy, whose coefficients belong to the algebra $B_{W}$ of functions from $\Gamma^{W}$ to $M_{m}(\mathbb{C})$ that are analytic w.r.t. each of the parameters $t_{k, i}$. For the derivation $\partial$ of $B_{W}$ we take $\sum_{1 \leq \alpha \leq m} \partial_{1, \alpha}$.

3.2. For each $\gamma \in \Gamma^{W}$, let $\hat{\psi}_{W}(\gamma)_{i}$ be the inverse image of $v_{0}^{(i)}$ under the natural projection $W \gamma^{-1} \rightarrow \mathbf{K}_{\geq 0}$. If the action of $\gamma^{-1}$ decomposes in $G_{n}$ as $\left(\begin{array}{ll}a & b \\ 0 & d\end{array}\right)$ and if $w=\left(\begin{array}{l}w_{+} \\ w_{-}\end{array}\right) \in \mathbf{P}$, then one verifies directly that

$$
\begin{aligned}
\hat{\psi}_{W}(\gamma)_{i} & =v_{0}^{(i)}+d w_{-} a^{-1}\left(a w_{+} a^{-1}+b w_{-} a^{-1}\right)^{-1}\left(v_{0}^{(i)}\right) \\
& =v_{0}^{(i)}+\sum_{k<0} \sum_{j=1}^{m} \alpha_{(k), i j}(\gamma) v_{k}^{(j)} .
\end{aligned}
$$


Since all the $\hat{\psi}_{W}(\gamma)_{i}$ belong to $W \gamma^{-1}$, we can define maps $\psi_{W, i}: \Gamma^{W} \rightarrow W$ by

$$
\psi_{W, i}(\gamma)=\left(\hat{\psi}_{W}(\gamma)_{i}\right) \gamma=\sum_{k \in \mathbb{Z}} \sum_{j=1}^{m} \beta_{(k), i j}(\gamma) v_{k}^{(j)} .
$$

From this formula and the form of the operators $a, b$, and $d$, it will be clear that all the maps $t_{j, i} \rightarrow \psi_{W, i}(\gamma)$, with $1 \leq i \leq m$ and $j \in \mathbb{N}$, are Fréchetdifferentiable. Next we introduce the matrices $\hat{\Psi}_{W}(\gamma)$ and $\Psi_{W}(\gamma)$ whose rows are the $\hat{\psi}_{W, i}(\gamma)$ and $\psi_{W, i}(\gamma)$, resp., $1 \leq i \leq m$. An essential property of $\Psi_{W}(\gamma)$ is that all its derivatives w.r.t. the $t_{i, j}$ have rows that all belong to $W$. We write $\alpha_{(k)}(\gamma)$, resp. $\beta_{(k)}(\gamma)$, for the matrices whose $(i, j)$ th entry is $\alpha_{(k), i j}(\gamma)$, resp. $\beta_{(k), i j}(\gamma)$; then $\alpha_{(k)}$ and $\beta_{(k)}$ belong to $B_{W}$. Following the lines set out in the first section, we introduce in $B_{W}\left[\xi, \xi^{-1}\right]$ the operators

$$
K_{W}=\mathrm{Id}+\sum_{k<0} \alpha_{(k)} \xi^{k}, \quad L_{W}=K_{W} \xi K_{W}^{-1}, \quad U_{\alpha}=L_{W} E_{\alpha} L_{W}^{-1},
$$

and we associate to $\hat{\Psi}_{W}(\gamma), \Psi_{W}(\gamma)$, and $\gamma \in \Gamma$, the power series $\hat{\Psi}_{W}[\lambda]$, $\Psi_{W}[\lambda]$, and $\gamma[\lambda]$ :

$$
\begin{gathered}
\hat{\Psi}_{W}[\lambda]=\mathrm{Id}+\sum_{k<0} \alpha_{(k)} \lambda^{k}, \quad \Psi_{W}[\lambda]=\sum_{k \in \mathbb{Z}} \beta_{(k)} \lambda^{k}, \\
\gamma[\lambda]=\exp \left(\sum_{k \geq 1} \operatorname{diag}\left(t_{k, 1}, \ldots, t_{k, m}\right) \lambda^{k}\right) .
\end{gathered}
$$

The action of $\Gamma$ is such that $\hat{\Psi}_{W}[\lambda], \Psi_{W}[\lambda]$, and $\gamma[\lambda]$ are related through $\Psi_{W}[\lambda]=\hat{\Psi}_{W}[\lambda] \gamma[\lambda]$, where the product is the usual one for the multiplication of power series. In other words, $\Psi_{W}[\lambda] \in M(\gamma[\lambda])$.

3.3. We want to show that $\Psi_{W}[\lambda]$ is a wavefunction corresponding to $L_{W}$ and the $U_{\alpha}$. Since $\partial_{k, \alpha} \gamma[\lambda]=\lambda^{k} E_{\alpha} \gamma[\lambda]$, we have $\partial_{k, \alpha}=\tilde{\partial}_{k, \alpha}$ on $M(\gamma[\lambda])$ and according to section (1.4), it suffices to prove

$$
\partial_{k, \alpha}\left(\Psi_{W}[\lambda]\right)=\left(L_{W}^{k} U_{\alpha}\right)_{+}\left(\Psi_{W}[\lambda]\right) \text {. }
$$

This relation is a consequence of the following

3.3.1. Proposition. For all $1 \leq \alpha \leq m$ and all $k \geq 1$ there exists a $P_{k, \alpha} \in B_{W}[\xi]$ of the form $P_{k, \alpha}=\sum_{j=0}^{k} P_{k, \alpha, j} \xi^{j}$, with $P_{k, \alpha, k}=E_{\alpha}$, such that

$$
\partial_{k, \alpha}\left(\Psi_{W}[\lambda]\right)=P_{k, \alpha}\left(\Psi_{W}[\lambda]\right) \text {. }
$$

If we substitute $\Psi_{W}[\lambda]=\hat{\Psi}_{W}[\lambda] \gamma[\lambda]$ in this relation we then get

$$
\left\{\partial_{k, \alpha}\left(\hat{\Psi}_{W}[\lambda]\right)+\hat{\Psi}_{W}[\lambda] \lambda^{k} E_{\alpha}\right\} \gamma[\lambda]=P_{k, \alpha}\left(\Psi_{W}[\lambda]\right) .
$$

By translating this back to $B_{W}\left[\xi, \xi^{-1}\right]$, we obtain the relation

$$
\partial_{k, \alpha} K_{W}+K_{W} \xi^{k} E_{\alpha}=P_{k, \alpha} K_{W},
$$

which implies $P_{k, \alpha}=\left(K_{W} \xi^{k} E_{\alpha} K_{W}^{-1}\right)_{+}=\left(L_{W}^{k} U_{\alpha}\right)_{+}$. 
Proof. First we have

$$
\begin{aligned}
\partial_{k, \alpha}\left(\Psi_{W}[\lambda]\right) & =\hat{\Psi}_{W}[\lambda] E_{\alpha} \lambda^{k} \gamma[\lambda]+\partial_{k, \alpha}\left(\hat{\Psi}_{W}[\lambda]\right) \gamma[\lambda] \\
& =\hat{\Psi}_{W}[\lambda] E_{\alpha} \lambda^{k} \gamma[\lambda]+\left(\sum_{i<0} c_{i} \lambda^{i}\right) \gamma[\lambda] \\
& =\left\{E_{\alpha} \lambda^{k}+\sum_{j<k} b_{j} \lambda^{j}\right\} \gamma[\lambda] .
\end{aligned}
$$

Next we consider the action of $\xi^{r}$ on $M(\gamma[\lambda])$. Here we look at the leading term modulo $\gamma[\lambda]$ :

$$
\begin{aligned}
\partial^{r}\left(\Psi_{W}[\lambda]\right) & =\sum_{j=0}^{r}\left(\begin{array}{l}
r \\
j
\end{array}\right) \partial^{j}\left(\hat{\Psi}_{W}[\lambda]^{r-j}\right) \gamma[\lambda] \\
& =\left(\operatorname{Id} \lambda^{r}+\sum_{j<r} d_{j} \lambda^{j}\right) \gamma[\lambda] .
\end{aligned}
$$

Hence there is an $s<k$ such that

$$
\partial_{k, \alpha}\left(\Psi_{W}[\lambda]\right)-E_{\alpha} \partial^{k}\left(\Psi_{W}[\lambda]\right)=\left(A_{s} \lambda^{s}+\sum_{j<s} \rho_{j} \lambda^{j}\right) \gamma[\lambda]
$$

Continuing in this way, after at most $k$ steps we obtain a polynomial $\sum_{i=0}^{k} A_{i} \partial^{i}$, with all the $A_{i}$ in $B_{W}$ and $A_{k}=E_{\alpha}$, satisfying

$$
\left(\partial_{k, \alpha}-\sum_{i=0}^{k} A_{i} \partial^{i}\right) \Psi_{W}[\lambda]=\left(\sum_{j<0} \mu_{j} \lambda^{j}\right) \gamma[\lambda] .
$$

Since multiplying a matrix from the left by another one involves only linear operations with the rows, all the rows of the $A_{i} \partial^{i}\left(\Psi_{W}(\gamma)\right)$ belong again to $W$. On the other hand, the rows of $\left(\partial_{k, \alpha}-\sum_{i=0} A_{i} \partial^{i}\right) \Psi_{W}(\gamma)$ also belong to $\left(\mathbf{K}_{<0}\right) \gamma$. Hence by the definition of $\Gamma^{W}$, they must be zero and we have the desired equality

$$
\left(\partial_{k, \alpha}-\sum_{i=0}^{k} A_{i} \xi^{i}\right) \Psi_{W}[\lambda]=0
$$

3.4. In this subsection we explain the connection between the scaling in (1.5) and that in (2.5). Take $c$ in $\mathbb{C}^{*}$ as in (2.5). If

$$
\lambda=\exp \left(\sum_{j=1}^{\infty} \operatorname{diag}\left(t_{j 1}, \ldots, t_{j m}\right) \underline{\Lambda}^{j}\right) \in \Gamma,
$$


then we write

$$
\begin{aligned}
\tilde{\gamma} & =\exp \left(\sum_{j \geq 1} \operatorname{diag}\left(c^{j} t_{j 1}, \ldots, c^{j} t_{j m}\right) \underline{\Lambda}^{j}\right) \\
& =\exp \left(\sum_{j \geq 1} \operatorname{diag}\left(t_{j 1}, \ldots, t_{j m}\right)(c \underline{\Lambda})^{j}\right) \\
& =\sum_{k \geq 0} c^{k} a_{k} \underline{\Lambda}^{k},
\end{aligned}
$$

with $a_{k} \in \operatorname{End}\left(\mathbb{C}^{m}\right)$. The relation needed is $R_{c} \tilde{\gamma} W=\gamma R_{c} W$ for all $\gamma \in \Gamma$ and all $W \in \operatorname{Gr}_{0}(\mathbf{K})$. Since each $\gamma=\sum_{i \geq 0} a_{i} \underline{\Lambda}^{i}=\lim _{n \rightarrow \infty} \sum_{i=0}^{n} a_{i} \underline{\Lambda}^{i}$, it suffices to prove this relation for $\gamma \in \Gamma$ of the form $\gamma=\sum_{i=1}^{k} a_{i} \Lambda^{i}$; then also $\tilde{\gamma}=\sum_{i=1}^{n} c^{i} a_{i} \underline{\Lambda}^{i}$. Now for $\underline{w}=\sum_{j \leq N} w_{j} \lambda^{j} \in W$, we have

$$
\begin{aligned}
R_{c} \tilde{\gamma}\left(\sum_{j \leq N} W_{j} \lambda^{j}\right) & =R_{c}\left(\sum_{i=1}^{n} \sum_{j \leq N} c^{i} a_{i}\left(w_{j}\right) \lambda^{i+j}\right) \\
& =\sum_{i=1}^{n} \sum_{j \leq N} c^{-j} a_{i}\left(w_{j}\right) \lambda^{i+j}, \\
\gamma R_{c}\left(\sum_{j \leq N} w_{j} \lambda^{j}\right) & =\sum_{i=1}^{n} \sum_{j \leq N} a_{i}\left(c^{-j} w_{j}\right) \lambda^{i+j}=R_{c} \tilde{\gamma} \underline{w} .
\end{aligned}
$$

The elements of $W$ of this form are dense in $W$, hence we get the desired equality. This implies

3.4.1. Proposition. The wave functions of $W$ and $R_{c} W$ are related through

$$
\hat{\Psi}_{R_{c} W}(\gamma)[\lambda] \gamma[\lambda]=\hat{\Psi}_{W}(\tilde{\gamma})[\lambda / c] \tilde{\gamma}[\lambda / c] \text {. }
$$

Proof. Since $\gamma[\lambda]=\tilde{\gamma}[\lambda / c]$ one merely has to show the equality for $\hat{\Psi}_{W}$ and $\hat{\Psi}_{R_{c} W}$. First we have $R_{c}\left(\hat{\Psi}_{W}(\tilde{\gamma})[\lambda]\right)=\hat{\Psi}_{W}(\tilde{\gamma})[\lambda / c]$. Thus the $i$ th row of $\hat{\Psi}_{W}(\tilde{\gamma})[\lambda / c]$ is the scaling by $c$ of the unique element of $\tilde{\gamma}^{-1} W$ of the form $f_{i}+\sum_{i<0}^{c} w_{j} \lambda^{j}$. In other words it is the unique element in $R_{c} \tilde{\gamma}^{-1} W=\gamma^{-1} R_{c} W$ of the form $f_{i}+$ lower order in $\lambda$ and that is exactly the $i$ th row of $\hat{\Psi}_{R_{c} W}(\gamma)[\lambda]$. This proves the proposition.

In particular, we see that $K_{R_{c} W}$ in $B\left[\xi, \xi^{-1}\right]$ is given by

$$
K_{R_{c} W}=\mathrm{Id}+\sum_{i>0} c^{i} \tilde{k}_{i} \xi^{-i}
$$

with $K_{W}=\mathrm{Id}+\sum_{i>0} k_{i} \xi^{-i}$. By making use of a grading argament as in (1.5), one shows that if $K_{W}^{-1}=\mathrm{Id}+\sum_{i>0} \underline{k}_{i} \xi^{-i}$, then

$$
K_{R_{c} W}^{-1}=\mathrm{Id}+\sum_{i>0} c^{i} \underline{\widetilde{K}}_{i} \xi^{-i} \text {. }
$$


Hence we get

\subsubsection{Proposition. $L_{R_{c} W}=K_{R_{c} W} \xi K_{R_{c} W}^{-1}=\widetilde{L}_{W}$ and $\widetilde{U}_{\alpha}=K_{R_{c} W} E_{\alpha} K_{R_{c} W}^{-1}$.}

Remark. Assuming that condition (2.5.1) holds for all sufficiently small $c$, we consider a $w$ in $\mathbf{P}$ with $\pi(w)=W_{k}$ such that $w\left(f_{\alpha} \lambda^{i}\right)=\sum_{k \leq i} \sum_{\beta=1}^{m} v_{k \beta} f_{\beta} \lambda^{k}$ for all $i \geq 0$ and $1 \leq \alpha \leq m$. Then we have that an embedding $R_{c} w$ corresponding to $R_{c} W$ is given by $R_{c} w\left(f_{\alpha} \lambda^{i}\right)=\sum_{k \leq i} \sum_{\beta=1}^{m} C^{i-k} v_{k \beta} f_{\beta} \lambda^{k}$. The coefficients $v_{k \beta}$ for $k \leq 0$ can be estimated by

$$
\left|v_{k \beta}\right|\left\|f_{\beta}\right\| \leq\left\|p_{\beta}\right\|\|\underline{\Lambda}\|^{-k}\|w\|\|\underline{\Lambda}\|^{i}
$$

where $p_{\beta}$ denotes the natural projection $K \rightarrow\left\langle f_{\beta}\right\rangle$. Hence given $p \geq 1$ one can apply a suitable small scaling by $c$ on $w$ such that $\mathbb{N}$-matrix corresponding to $R_{c} w$ has all columns in $l^{p}(\mathbb{Z})^{m}$. In this sense, the convergent power series solution of (1.4.1)-(1.4.3) to $K$ can be scaled into a solution corresponding to $l^{p}(\mathbb{Z})^{m}$.

\section{RELATION WITH $\tau$-FUNCTIONS}

4.1. Supposing that $W \in \mathrm{Gr}$, we will introduce some other elements of $\mathrm{Gr}$ closely related to $W$. In 4.3 we will need these elements to find a relation between the wave function $\Psi_{W}[\lambda]$ and some $\tau$-functions in the case $W=\mathbf{O}$.

We will need $\Lambda: H \rightarrow H$ to be invertible, so we require that $\Lambda: H \rightarrow H$ be surjective. By the bounded inverse theorem, $\Lambda^{-1}$ is also bounded.

Let $\Lambda_{k_{1}, \ldots, k_{m}}$ be the automorphism of $\mathbf{K}=\bigoplus_{i=1}^{m} H^{(i)}$ of the form $\operatorname{diag}\left(\Lambda^{k_{1}}, \ldots, \lambda^{k_{m}}\right)$, and $W_{k_{1}, \ldots, k_{m}}=W \cdot \Lambda_{k_{1}, \ldots, k_{m}}$. Now $W_{k_{1}, \ldots, k_{m}}$ is again an element of $\mathrm{Gr}$ if $\Lambda_{k_{1}, \ldots, k_{m}}$ is an element of $G_{n}$, and this is the case when $k_{1}+\cdots+k_{m}=0$.

As is easily verified, $\left(\sum a_{1 i} v_{i+k_{1}}, \ldots, \sum a_{m i} v_{i+k_{m}}\right) \in W_{k_{1}, \ldots, k_{m}}$ if and only if $\left(\sum a_{1 i} v_{i}, \ldots, \sum a_{m i} v_{i}\right) \in W=W_{0, \ldots, 0}$.

In the sequel we are interested only in the case where most of the $k_{i}$ are zero. Therefore we introduce $\Lambda_{i / j}=\Lambda_{k_{1}, \ldots, k_{m}}$ for $i \neq j$, with $k_{i}=1, k_{j}=-1$, $k_{l}=0 \quad(l \neq i, j)$. We denote $W_{i / j}=W \Lambda_{i / j}$ correspondingly.

Now we want to extend the elements $\Lambda_{i / j} \in G_{n}$ to elements $\left(\Lambda_{i / j}, q_{i / j}\right) \in G$. To perform this extension, we consider the splitting

$$
\Lambda_{i / j}=\left(\begin{array}{cc}
a_{i / j} & b_{i} \\
c_{j} & d_{i / j}
\end{array}\right)
$$

with respect to the direct sum $\mathbf{K}=\mathbf{K}_{\geq 0} \oplus \mathbf{K}_{<0}$. As mentioned before, $a_{i / j}: \mathbf{K}_{\geq 0}$ $\rightarrow \mathbf{K}_{\geq 0}$ is a Fredholm operator of index zero. In fact $\operatorname{ker}\left(a_{i / j}\right)=\mathbb{C} v_{0}^{(j)}=\mathbb{C} e_{j-1}$ and $\operatorname{coker}\left(a_{i / j}\right) \cong \mathbb{C} v_{0}^{(i)}=\mathbb{C} e_{i-1}$. We introduce the operator $E_{k, l}$ mapping $\sum \alpha_{i} e_{i}$ to $\alpha_{l} e_{k}$. Then $q_{i / j}=a_{i / j}+E_{i-1, j-1}$ is invertible, moreover $q_{i / j}^{-1} a_{i / j}=$ id $-E_{j-1, j-1}$, so it is surely of the form id + nuclear. Finally notice that $b_{i}=$ $E_{i-1, i-1-m}$ is an operator $\mathbf{K}_{<0} \rightarrow \mathbf{K}_{\geq 0}$. 
4.2. To introduce $\tau$-functions, we have to fix an embedding $w \in \mathbf{P}$ such that $\operatorname{im}(w)=W . \tau_{w}: G \rightarrow \mathbb{C}$ is defined in 2.4. Now suppose $\tau_{w}\left(g_{1}\right) \neq 0$. Then using the multiplicativity of the determinant one can prove

$$
\frac{\tau_{w}\left(g_{1} g_{2}\right)}{\tau_{w}\left(g_{1}\right)}=\tau_{\left(g_{1}^{-1} w\right) \cdot\left(g_{1}^{-1} w\right)_{+}^{-1}}\left(g_{2}\right),
$$

Notice that $w_{1}=\left(g_{1}^{-1} w\right) \circ\left(g_{1}^{-1} w\right)_{+}^{-1}$ is of the form $\left(\begin{array}{c}\text { id } \\ \left(w_{1}\right)_{-}\end{array}\right)$. We apply this formula with $g_{1} \in \Gamma^{W}$, and

$$
g_{2}=\operatorname{diag}\left(\mathrm{id}, \ldots, \mathrm{id}, q_{\zeta}, \mathrm{id}, \ldots, \mathrm{id}\right)=q_{\zeta}^{(i)},
$$

where $q_{\zeta}=\mathrm{id}-\Lambda \zeta^{-1}$, is placed in the $(i, i)$-entry of $g_{2}$. We take $|\zeta|>\|\Lambda\|$ to ensure that $q_{\zeta} \in \Gamma(\Lambda)$ and $q_{\zeta}^{(i)} \in \Gamma$.

In the 1-component case it is proved that upon splitting $q_{\zeta}^{-1}=\left(\begin{array}{ll}a & b \\ 0 & d\end{array}\right)$ with respect to $H_{\geq 0} \oplus H_{<0}$, one has that $a^{-1} b: H_{<0} \rightarrow H_{\geq 0}$ is the 1-dimensional projection of $H_{<0}$ onto $H_{0}$ given by

$$
a^{-1} b\left(\sum_{j<0} a_{j} v_{j}\right)=\left(\sum_{j<0} a_{j} \zeta^{j}\right) v_{0} \quad(\text { see }[\text { Se or He] }) .
$$

From this one easily derives that if

$$
q_{\zeta}^{(i)^{-1}}=\left(\begin{array}{cc}
a^{(i)} & b^{(i)} \\
0 & d^{(i)}
\end{array}\right)
$$

with respect to $\mathbf{K}_{\geq 0} \oplus \mathbf{K}_{<0}$, then $a^{(i)^{-1}} b^{(i)}: \mathbf{K}_{<0} \rightarrow \mathbf{K}_{\geq 0}$ is the 1-dimensional projection on $\mathbf{K}_{i-1}=\mathbb{C} e_{i-1}=\mathbb{C} v_{0}^{(i)}$ given by

$$
a^{(i)^{-1}} b^{(i)}\left(\sum_{j<0} a_{1 j} v_{j}, \ldots, \sum_{j<0} a_{m j} v_{j}\right)=\left(\sum_{j<0} a_{i j} \zeta^{j}\right) v_{0}^{(i)} .
$$

Now it is easy to derive the following

Lemma. Suppose $\gamma \in \Gamma^{W}$ and $\operatorname{Im}(w)=W, w \in \mathbf{P}$. Then

$$
\Psi_{W,}[\zeta]_{i i}=1+\sum_{k<0} \alpha_{(k) i i} \zeta^{k}=\frac{\tau_{w}\left(\gamma q_{\zeta}^{(i)}\right)}{\tau_{w}(\gamma)} .
$$

Proof. We have to calculate

$$
\begin{aligned}
\tau_{w_{1}}\left(q_{\zeta}^{(i)}\right) & =\operatorname{det}\left(\left(a^{(i)}+b^{(i)}\left(w_{1}\right)_{-}\right) a^{(i)^{-1}}\right) \\
& =\operatorname{det}\left(\operatorname{id}+a^{(i)^{-1}} b^{(i)}\left(w_{1}\right)_{-}\right) \\
& =1+\operatorname{trace}\left(a^{(i)^{-1}} b^{(i)}\left(w_{1}\right)_{-}\right) .
\end{aligned}
$$


Since

$$
\left(w_{1}\right)_{-}\left(e_{i-1}\right)=\left(w_{1}\right)_{-}\left(v_{0}^{(i)}\right)=\left(\sum_{k<0} \alpha_{(k) i 1} v_{k}, \ldots, \sum_{k<0} \alpha_{(k) i m} v_{k}\right),
$$

we have the desired result by using the explicit form of $a^{(i)^{-1}} b^{(i)}$.

4.3. The results of the last lemma enable us to express the diagonal elements of $\hat{\Psi}_{W}[\lambda]$ in terms of the function $\tau_{w}$. For the off-diagonal element $\hat{\Psi}_{W}[\lambda]_{j i}$, $j \neq i$, we need the space $W_{i / j}$. We fix $w \in \mathbf{P}$ such that $\pi(w)=W$. Further we introduce $w_{i / j}=\Lambda_{i / j} w q_{i / j}^{-1}$. We will express $\hat{\Psi}_{W}[\lambda]_{j i}$ in terms of $\tau_{w_{i / j}}$ and $\tau_{w}$. First we will prove this expression in the case $a_{(-1) i j} \neq 0$. This is an extra condition on $\gamma \in \Gamma^{W}$. This derivation shows how one comes to this expression. Finally we give a derivation which also holds when $a_{(-1) j i}=0$.

To find $\Psi_{W}[\lambda]_{j i}$ in terms of $\tau_{w_{i / j}}$ we consider the complete row $\psi_{w}(\gamma)_{j}$ :

$$
\left(\sum_{k<0} \alpha_{(k) j 1} v_{k}, \ldots, v_{0}+\sum_{k<0} \alpha_{(k) j j} v_{k}, \ldots, \sum_{k<0} \alpha_{(k) j m} v_{k}\right) \in W \gamma^{-1} .
$$

Multiplying by $\Lambda_{i / j}$ we find

$$
\begin{aligned}
& \alpha_{(-1) j i}\left(\sum_{k<0} \frac{\alpha_{(k) j 1}}{\alpha_{(-1) j i}} v_{k}, \ldots, v_{0}+\sum_{k<-1} \frac{\alpha_{(k) j i}}{\alpha_{(-1) j i}} v_{k+1}, \ldots, \sum_{k<0} \frac{\alpha_{(k) j m}}{\alpha_{(-1) j i}} v_{k}\right) \\
& \in W \gamma^{-1} \Lambda_{i / j}=W_{i / j} \gamma^{-1} .
\end{aligned}
$$

Because we required $\alpha_{(-1) j i} \neq 0$, the natural projection $W_{i / j} \gamma^{-1} \rightarrow \mathbf{K}_{\geq 0}$ is a bijection. Using the results of 4.2 we find that

$$
1+\sum_{k<-1} \frac{\alpha_{(k) j i}}{\alpha_{(-1) j i}} \zeta^{k+1}=\frac{\tau_{w_{i / j}}\left(\gamma q_{\zeta}^{(i)}\right)}{\tau_{w_{i / j}}(\gamma)} .
$$

So we obtain the following result:

$$
\hat{\Psi}_{W}[\zeta]_{j i}=\zeta^{-1} \alpha_{(-1) j i} \frac{\tau_{w_{i / j}\left(\gamma q_{\zeta}^{(i)}\right)}}{\tau_{w_{i / j}(\gamma)}} .
$$

Now it is easy to prove the following theorem in the case $\alpha_{(-1) j i} \neq 0$.

Theorem. Suppose $\gamma \in \Gamma^{W}$ and define $\hat{\Psi}_{W}(\gamma)$ as before. Then

$$
\hat{\Psi}_{W}[\zeta]_{j i}=\zeta^{-1} \frac{\tau_{w_{i / j}}\left(\gamma q_{\zeta}^{(i)}\right)}{\tau_{w}(\gamma)}, \quad j \neq i .
$$

Proof. For convenience we write $A=\left(\gamma^{-1} w a^{-1}\right)_{+}^{-1} \in \mathbf{T}$, where $\gamma^{-1}=\left(\begin{array}{ll}a & b \\ 0 & d\end{array}\right)$. Then $\tau_{w}(\gamma)=\operatorname{det}\left(\left(\gamma^{-1} w a^{-1}\right)_{+}\right)=\operatorname{det} A^{-1}$. 
On the other hand, since $\operatorname{det}\left(a q_{i / j} a^{-1} q_{i / j}^{-1}\right)=1$,

$$
\begin{aligned}
\tau_{w_{i / j}}(\gamma) & =\operatorname{det}\left(\left(\gamma^{-1} \Lambda_{i / j} w\right)_{+} q_{i / j}^{-1} a^{-1}\right) \\
& =\operatorname{det}\left(\left(\Lambda_{i / j} \gamma^{-1} w a^{-1}\right)_{+} q_{i / j}^{-1}\right) \\
& =\operatorname{det}\left(A^{-1} q_{i / j}^{-1}\left(\Lambda_{i / j} \gamma^{-1} w a^{-1} A\right)_{+}\right) \\
& =\operatorname{det}\left(A^{-1}\right) \cdot \operatorname{det}\left(q_{i / j}^{-1} a_{i / j}+q_{i / j}^{-1} b_{i}\left(w_{1}\right)_{-}\right) .
\end{aligned}
$$

So $\tau_{w_{i / j}}(\gamma)=\operatorname{det}\left(q_{i / j}^{-1} a_{i / j}+q_{i / j}^{-1} b_{i} w_{-}\right) \cdot \tau_{w}(\gamma)$.

Now $q_{i / j}^{-1} a_{i / j}=\mathrm{id}-E_{j-1, j-1}$ and $q_{i / j}^{-1} b_{i}$ is the projection $E_{j-1, i-1-m}$. So $q_{i / j}^{-1} a_{i / j}+q_{i / j}^{-1} b_{i}\left(w_{1}\right)_{-}$is of the form id +1 -dimensional, so we are left with the calculation of

$$
1+\operatorname{trace}\left(-E_{j-1, j-1}+E_{j-1, i-1-m}\left(w_{1}\right)_{-}\right) .
$$

Since $\left(-E_{j-1, j-1}+E_{j-1, i-1-m}\left(w_{1}\right)_{-}\right)\left(e_{j-1}\right)=\left(-1+\alpha_{(-1) j i}\right)\left(e_{j-1}\right)$ we find $\tau_{w_{i / j}}(\gamma)=\alpha_{(-1) j i} \cdot \tau_{w}(\gamma)$, proving the result.

We now turn to a proof for the last theorem without assuming $\alpha_{(-1) j i} \neq 0$. We define two elements of $\mathbf{P}$, namely $w_{1}$ (as above) and $v_{i / j}$ :

$$
\begin{gathered}
w_{1}=\left(\gamma^{-1} w a^{-1}\right)\left(\gamma^{-1} w a^{-1}\right)_{+}^{-1}=\left(\begin{array}{c}
\mathrm{id} \\
\left(w_{1}\right)_{-}
\end{array}\right), \\
v_{i / j}=\Lambda_{i / j} w_{1} q_{i / j}^{-1} .
\end{gathered}
$$

Now we have

Lemma. $\tau_{w_{i / j}}\left(\gamma q_{\zeta}^{(i)}\right)=\tau_{w}(\gamma) \tau_{v_{i / j}}\left(q_{\zeta}\right)$.

Proof.

$$
\begin{aligned}
\tau_{w_{i / j}}\left(\gamma q_{\zeta}^{(i)}\right) & =\operatorname{det}\left(\left(q_{\zeta}^{(i)^{-1}} \gamma^{-1} \Lambda_{i / j} w q_{i / j}^{-1} a^{-1} a^{(i)^{-1}}\right)_{+}\right) \\
& =\operatorname{det}\left(\left(q_{\zeta}^{(i)^{-1}} \Lambda_{i / j} \gamma^{-1} w a^{-1} q_{i / j}^{-1} a^{(i)^{-1}}\right)_{+}\right) \\
& =\tau_{w}\left(\gamma\left(\tilde{\Lambda}_{i / j}^{-1} q_{\zeta}^{(i)}\right)\right) .
\end{aligned}
$$

Here we used that $\Lambda_{i / j}$ and $\gamma^{-1}$ commute, and that $\operatorname{det}\left(a q_{i / j} a^{-1} q_{i / j}^{-1}\right)=1$. Further $\tilde{\Lambda}_{i / j}^{-1}=\left(\Lambda_{i / j}, q_{i / j}\right)^{-1}$. Using the multiplicity formula (4.2.1) we find

$$
\tau_{w}\left(\gamma\left(\tilde{\Lambda}_{i / j}^{-1} q_{\zeta}^{(i)}\right)\right)=\tau_{w}(\gamma) \cdot \tau_{w_{1}}\left(\tilde{\Lambda}_{i / j}^{-1} q_{\zeta}^{(i)}\right)=\tau_{w}(\gamma) \cdot \tau_{v_{i / j}}\left(q_{\zeta}^{(i)}\right) .
$$

It is left to prove that $\tau_{v_{i / j}}\left(q_{\zeta}^{(i)}\right)=\zeta \tilde{\Psi}_{W}[\zeta]_{j i}$. So let us investigate $v_{i / j}=\left(\begin{array}{l}v_{+} \\ v_{-}\end{array}\right)$, with

$$
\begin{aligned}
v_{+} & =a_{i / j} q_{i / j}^{-1}+b_{i}\left(w_{1}\right)_{-} q_{i / j}^{-1} \\
& =\left(\mathrm{id}-E_{i-1, i-1}\right)+b_{i}\left(w_{1}\right)_{-} q_{i / j}^{-1} \\
& =\mathrm{id}+\operatorname{proj} \text { on } \mathbb{C} e_{i-1} .
\end{aligned}
$$


Moreover $\tau_{v_{i / j}}\left(q_{\zeta}^{(i)}\right)=\operatorname{det}\left(v_{+}+a^{(i)^{-1}} b^{(i)} v_{-}\right)$. Now $a^{(i)^{-1}} b^{(i)}$ is also a projection on $\mathbb{C} e_{i-1}$. So

$$
\begin{aligned}
\operatorname{det} & \left.v_{+}+a^{(i)^{-1}} b^{(i)} v_{-}\right) \\
& =1+\operatorname{trace}\left(-E_{i-1, i-1}+b_{i}\left(w_{1}\right)_{-} q_{i / j}^{-1}+a^{(i)^{-1}} b^{(i)} v_{-}\right) \\
& =1+(-1+s(\zeta))=s(\zeta)
\end{aligned}
$$

where $s(\zeta)$ is given by

$$
\left(b_{i}\left(w_{1}\right)_{-} q_{i / j}^{-1}+a^{(i)^{-1}} b^{(i)} v_{-}\right)\left(e_{i-1}\right)=s(\zeta) e_{i-1} .
$$

Now $b_{i}\left(w_{1}\right)-q_{i / j}^{-1}\left(e_{i-1}\right)=\alpha_{(-1) j i} e_{i-1}$ and

$$
\left(a^{(i)^{-1}} b^{(i)} v_{-}\right)\left(e_{i-1}\right)=\left(\sum_{k<-1} \alpha_{(k) j i} \zeta^{k+1}\right) e_{i-1},
$$

which proves the theorem in the general case.

4.4. Let us discuss these results. First notice that the spaces $W_{k_{1}, \ldots, k_{m}}$ in [U] are what we called $W_{-k_{1}, \ldots,-k_{m}}$, which can be seen by comparing the expression of $\hat{\Psi}_{W}$ in $\tau_{w}$ and $\tau_{w_{k_{1}, \ldots, k_{m}}}$ in both cases.

Second we want to consider $\tau_{w}$ as a function of variables $\left(t_{j, \alpha}\right), j=$ $1,2, \ldots, \alpha=1, \ldots, m$. Therefore we write

$$
\gamma^{-1}=\operatorname{diag}\left(\exp \left(\sum_{j \geq 1} t_{j, 1} \Lambda^{j}\right), \ldots, \exp \left(\sum_{j \geq 1} t_{j, m} \Lambda^{j}\right)\right),
$$

and denote the set of $\left(t_{j, \alpha}\right)$ for which $\gamma^{-1} \in \Gamma$ by $T$, and $\tau_{w}(\gamma)$ by $\tau_{w}\left(\left(t_{j, \alpha}\right)\right)$. In the case that $\left(t_{j, \alpha}\right) \in T$, we have the following assertions:

$1^{\circ} \tau_{w}\left(\left(t_{j, \alpha}\right)\right)$ is well defined.

$2^{\circ} \tau_{w}\left(\left(t_{j, \alpha}\right)\right)$ is a globally defined analytic function (of any finite number of variables).

This last statement can be proved using the expression of the determinant in traces of exterior powers of $\left(\gamma^{-1} w a^{-1}\right)_{+}-\mathrm{id}$ (see [He]). To prove that $T \neq \varnothing$, note that $\left(t_{j, \alpha}\right)$ with only finitely many $t_{j, \alpha}$ nonzero are elements of $T$.

All this has consequences for the wave function $\hat{\Psi}_{W}[\zeta]$. Since

$$
\begin{aligned}
\left(\gamma q_{\zeta}^{(i)}\right)^{-1}=\operatorname{diag}( & \exp \left(\sum t_{j, 1} \Lambda^{j}\right), \ldots, \\
& \left.\quad \exp \left(\sum\left(t_{j, i}-\frac{1}{j \zeta}\right) \Lambda^{j}\right), \ldots, \exp \left(\sum t_{j, m} \Lambda^{j}\right)\right)
\end{aligned}
$$


we see that for $\tau_{w}(\gamma) \neq 0, \hat{\Psi}_{W}[\zeta]_{j i}$ is an analytic function of (any finite number of) $\left(t_{j, \alpha}\right)$ and $\zeta$ for $|\zeta|>\|\Lambda\|$.

\section{REDUCTIONS}

In this section we consider some reductions of the multicomponent KPhierarchy. First in 5.1 we show that very general Lax equations, as considered in [Wi], are obtained as reductions. Moreover in subsection 5.2 we show that an easy case of the reductions leads to the AKNS-hierarchy, as defined in [FNR].

Throughout this section,

$$
\underline{\Lambda}^{l}=\Lambda_{l, \ldots, l}=\operatorname{diag}\left(\Lambda^{l}, \ldots, \Lambda^{l}\right) .
$$

5.1. Suppose that $W \in \mathrm{Gr}$ satisfies

$$
w \in W \Rightarrow \sum_{\beta=1}^{m} c_{\beta} w E_{\beta} \underline{\Lambda}^{l} \in W \quad\left(c_{\beta} \in \mathbb{C}\right) .
$$

A special case of this reduction is $c_{\beta}=1$ leading to

$$
w \in W \Rightarrow \sum_{\beta=1}^{m} w E_{\beta} \underline{\Lambda}^{l}=w \underline{\Lambda}^{l} \in W .
$$

We denote $\mathscr{L}_{w}=\sum_{\beta=1}^{m} c_{\beta} L_{w}^{l} U_{\beta}$. We prove the following.

Assertion. $W \in \mathrm{Gr}$ satisfies $(5.1 .1) \Rightarrow \mathscr{L}_{w}=\left(\mathscr{L}_{w}\right)_{+}$.

Proof (we write $\mathscr{L}$ instead of $\mathscr{L}_{w}$ ).

$$
\begin{aligned}
\sum_{\beta} c_{\beta} P_{l, \beta}\left(\psi_{w}[\lambda]\right) & \\
= & \sum_{\beta} c_{\beta} \partial_{l, \beta}\left(\left(\sum_{k \leq 0} \alpha_{(k)} \lambda^{k}\right) \gamma[\lambda]\right) \\
& =\left(\sum_{\substack{k \leq-1 \\
\beta}} c_{\beta} \partial_{l, \beta}\left(\alpha_{(k)}\right) \lambda^{k}+\sum_{k \leq 0} c_{\beta} \alpha_{(k)} \lambda^{k} \lambda^{l} E_{\alpha}\right) \gamma[\lambda],
\end{aligned}
$$

so

$$
\begin{gathered}
\sum_{\beta} c_{\beta} P_{l, \beta} \psi_{w}[\lambda]-\lambda^{l} \sum_{\beta} c_{\beta} \psi_{w}[\lambda] E_{\beta} \\
=\left(\sum_{\substack{k \leq-1 \\
\beta}} c_{\beta} \partial_{l, \beta}\left(\alpha_{(k)}\right) \lambda^{k}\right) \gamma[\lambda] .
\end{gathered}
$$


Because of (5.1.1), $\lambda^{l} \sum_{\beta} c_{\beta} \psi_{w}[\lambda] E_{\beta} \in W$ and with it the left-hand side of (5.1.2), so the right-hand side, is a member of $W \cap \mathbf{K}_{\leq 0} \cdot \gamma=\{0\}$. Therefore

$$
\begin{gathered}
\sum_{\beta} c_{\beta} P_{l, \beta} \psi_{w}[\lambda]=\lambda^{l} \sum_{\beta} c_{\beta} \psi_{w}[\lambda] E_{\beta}, \\
\sum_{\beta} c_{\beta} \partial_{l, \beta} \alpha_{(k)} E_{\beta}=0 \quad(\forall k \leq 0) .
\end{gathered}
$$

Translating these equations back to $B\left[\xi, \xi^{-1}\right]$ and writing $\widehat{W}=K_{W}$, the first one yields

$$
\mathscr{L}_{+} \widehat{W}=\sum_{\beta} c_{\beta} \widehat{W} \xi^{l} E_{\beta},
$$

and the second one becomes

$$
\sum_{\beta} c_{\beta} \partial_{l, \beta}(\widehat{W}) E_{\beta}=0 .
$$

On the other hand we have $L^{l} \widehat{W}=\widehat{W} \xi^{l}$, from which we derive

$$
\begin{aligned}
\sum_{\beta} c_{\beta} \widehat{W} \xi^{l} E_{\beta} & =\sum_{\beta} c_{\beta} L^{l} \widehat{W} E_{\beta} \\
& =\sum_{\beta} c_{\beta} L^{l} U_{\beta} \widehat{W}=\mathscr{L} \widehat{W} .
\end{aligned}
$$

Comparing with $(5.1 .3)$ yields $\mathscr{L}_{+}=\mathscr{L}$, since $\widehat{W}$ is invertible. This proves the assertion.

$\mathscr{L}$ is a differential polynomial $\sum_{i=0}^{l} q_{i} \xi^{i}$ with leading terms

$$
\mathscr{L}=\sum_{\beta=1}^{m} c_{\beta} E_{\beta} \xi^{l}+\sum_{\beta=1}^{m} c_{\beta}\left[\alpha_{-1}, E_{\beta}\right] \xi^{l-1}+\cdots .
$$

Therefore $q_{l}=\operatorname{diag}\left(c_{1}, c_{2}, \ldots, c_{m}\right)$ and $\left(q_{l-1}\right)_{i j}=\left(\alpha_{-1}\right)_{i j}\left(c_{i}-c_{j}\right)$. In particular, $\left(q_{l-1}\right)_{i j}=0$ if $c_{i}=c_{j}$, so $\mathscr{L}$ is of the form studied by Wilson in [Wi]. From the equations for $L$ one easily derives

$$
\partial_{i, \beta}(\mathscr{L})=\left[\left(L^{i} U_{\beta}\right)_{+}, \mathscr{L}\right] .
$$

This yields partial differential equations for the matrix elements $\left(\alpha_{k}\right)_{i j}$ of $\widehat{W}$. In [Wi] conditions are discussed such that one finds equations expressed in the elements $\left(q_{s}\right)_{i j}$ of $\mathscr{L}$ only. This amounts to restricting oneself to certain derivations of the form $\sum_{\beta=1}^{m} d_{\beta} \partial_{i, \beta}$ such that the leading term of $\sum_{\beta=1}^{m} d_{\beta} L^{i} U_{\beta} \quad(\in Z(\mathscr{L}))$ is admissible (see Definition 2.17 of [Wi]), that is $d_{\alpha}=d_{\beta}$ if $c_{\alpha}=d_{\beta}$. 
5.2. We consider a special case of the reduction of 5.1, namely we take $c_{\beta}=1$ $(\beta=1, \ldots, m)$. In this case $\mathscr{L}=L^{l}$. Moreover from (5.1.4) one finds $\sum_{\beta} \partial_{l, \beta}(\widehat{W})=0$. In particular, for $l=1$ we have $\partial(\widehat{W})=0$. Since $L=$ $\widehat{W} \xi \widehat{W}^{-1}$ and $U_{\alpha}=\widehat{W} E_{\alpha} \widehat{W}^{-1}$ we also have

$$
\sum_{\beta} \partial_{l, \beta}(L)=\sum_{\beta} \partial_{l, \beta}\left(U_{\alpha}\right)=0 .
$$

Next we consider the easiest case of the situation above, namely we take $m=2$ and $l=1$, which leads to

$$
\mathscr{L}=L=\left(\begin{array}{ll}
1 & 0 \\
0 & 1
\end{array}\right) \xi
$$

We have two series of equations, (1.3.1) and (1.3.2). As noted, $\partial\left(L^{i} U_{\alpha}\right)_{+}=0$ so (1.3.1) is trivially satisfied. We rewrite (1.3.2) in the following form:

$$
\begin{aligned}
& \left(\partial_{i, 1}+\partial_{i, 2}\right) U_{\beta}=\left[\left(L^{i}\left(U_{1}+U_{2}\right)\right)_{+}, U_{\beta}\right], \\
& \left(\partial_{i, 1}-\partial_{i, 2}\right) U_{\beta}=\left[\left(L^{i}\left(U_{1}-U_{2}\right)\right)_{+}, U_{\beta}\right] .
\end{aligned}
$$

Now $\left[\left(L^{i}\left(U_{1}+U_{2}\right)\right)_{+}, U_{\beta}\right]=\left[\operatorname{Id} \cdot \xi, U_{\beta}\right]=0$, so $\left(\partial_{i, 1}+\partial_{i, 2}\right) U_{\beta}=0$. This means that $U_{\beta}$ depends only on $x_{i}=\frac{1}{2}\left(t_{i, 1}-t_{i, 2}\right)$, and not on $y_{i}=\frac{1}{2}\left(t_{i, 1}+t_{i, 2}\right)$. Therefore denote $\partial_{i}=\partial / \partial x_{i}=\partial_{i, 1}-\partial_{i, 2}$, so we have

$$
\partial_{i}\left(U_{\beta}\right)=\left[\left(L^{i}\left(U_{1}-U_{2}\right)\right)_{+}, U_{\beta}\right],
$$

which is equivalent to

$$
\begin{aligned}
& \partial_{i}\left(U_{1}+U_{2}\right)=\left[\left(\xi^{i} Q\right)_{+}, U_{1}+U_{2}\right], \\
& \partial_{i}\left(U_{1}-U_{2}\right)=\left[\left(\xi^{i} Q\right)_{+}, U_{1}-U_{2}\right] .
\end{aligned}
$$

We denoted $Q=U_{1}-U_{2}$. Since $U_{1}+U_{2}=\mathrm{Id},(5.2 .5)$ is trivial. We are left with

$$
\partial_{i} Q=\left[\left(\xi^{i} Q\right)_{+}, Q\right] .
$$

These are the equations of the AKNS-hierarchy, as defined by Flaschka, Newell, and Ratiu in [FNR]. Note that $\xi$ takes the role of spectral parameter (it commutes with $Q$ ). One can show that

$$
Q=\left(\begin{array}{cc}
1 & 0 \\
0 & -1
\end{array}\right)+\left(\begin{array}{cc}
0 & q \\
r & 0
\end{array}\right) \xi^{-1}+\cdots
$$

and all equations (5.2.7) can be expressed in $q$ and $r$. Moreover $Q^{2}=$ $\left(U_{1}-U_{2}\right)\left(U_{1}-U_{2}\right)=$ Id .

5.3. To give an idea of what is going on, we associate groups to these reductions. We denote

$$
\begin{aligned}
\Omega & =\operatorname{diag}\left(c_{1} \Lambda^{l}, c_{2} \Lambda^{l}, \ldots, c_{m} \Lambda^{l}\right) \\
& =\sum_{k \in \mathbb{Z}} c_{k+1} E_{k, k+l m} \quad\left(c_{\alpha+m}=c_{\alpha}\right) .
\end{aligned}
$$


Expressed in $\Omega$, (5.1.1) becomes $\omega \in W \Rightarrow \omega \Omega \in W$. Consider now the subgroup $G_{\Omega}$ of $G_{n}$ consisting of the elements of $G_{n}$ that commute with $\Omega$ :

$$
G_{\Omega}=\left\{g \in G_{n} \mid g \Omega=\Omega g\right\} .
$$

We consider the orbit $G_{\Omega} \cdot \mathbf{K}_{\geq 0}$ in Gr. One directly verifies that elements from this orbit satisfy (5.1.1). We describe $G_{\Omega}$ explicitly. For this we consider the matrix $\left(g_{i j}\right)$ of $g$ defined by $g \cdot e_{j}=\sum_{i} g_{i j} e_{i}$. Since $e_{j}$ is a topological basis of $\mathbf{K}, g$ is completely determined by its matrix. Condition (5.3.1) now yields

$$
g \in G_{\Omega} \Leftrightarrow g_{i, j-l m} c_{j+1}=g_{i+l m, j} c_{i+1} \quad(\forall i, j \in \mathbb{Z}) .
$$

A case of special interest is the case $c_{\beta}=1(\beta=1, \ldots, m)$. Then (5.3.2) gives

$$
g \in G_{\Omega} \Leftrightarrow g_{i, j}=g_{i+l m, j+l m} \quad(\forall i, j \in \mathbb{Z}) .
$$

Let us take $\mathbf{K}=l^{2}(\mathbb{Z}) \simeq L^{2}\left(S^{1}\right)$ and denote by $\mathrm{GL}_{P}(C)$ the group of twice differential maps $S^{1} \rightarrow \mathrm{GL}_{P}(\mathbb{C})$. Then using (5.3.3) and Proposition 2.3 of [Se] we see that there is an embedding of $\mathrm{GL}_{l m}(\mathbb{C})$ into $G_{\Omega} \subset G_{n}$.

Conversely, one can define $\mathrm{Gr}^{(l m)}$ in $l^{2}(\mathbb{Z})$ to be the $\mathrm{GL}_{l m}(\mathbb{C})$-orbit of $\mathbf{K}_{\geq 0}$ in the $m$-component KP-hierarchy. In this way we associate to $\mathrm{GL}_{P}(\mathbb{C})$ as many hierarchies as $p$ has positive divisors. In the case $p=2$ one finds two hierarchies: the KdV-hierarchy $m=1, l=2$ (see [Se]), and the AKNShierarchy $m=2, l=1$ (see 5.2).

\section{REFERENCES}

[FNR] H. Flaschka, A. C. Newell, and T. Ratiu, Kac-Moody Lie algebras and soliton equations, Phys. D 9 (1983), 300-323.

[He] G. F. Helminck, Convergent solutions of the KP-hierarchy, Memorandum 620 (April 1987), University of Twente.

[Se] G. B. Segal and G. Wilson, Loop groups and equations of KdV type, Inst. Hautes Études Sci. Publ. Math. 61 (1985), 5-65.

[U] K. Ueno and K. Takasaki, Toda-lattice hierarchy, Publ. Res. Inst. Math. Sci. 423 (1983).

[Wi] G. Wilson, Commuting flows and conservation laws for Lax equations, Math. Proc. Cambridge Philos. Soc. 86 (1979), 131-143.

Faculty of Applied Mathematics, University of Twente, P.O. Box 217, 7500 AE ENSCHEDE, The NeTHERLANDS 